\title{
EXAMPLES OF NONNORMAL SEMINORMAL OPERATORS WHOSE SPECTRA ARE NOT SPECTRAL SETS
}

\begin{abstract}
KEVIN F. CLANCEY
Abstract. An example is given of a nonnormal seminormal operator on a Hilbert space whose spectrum is thin (in the sense of von Neumann) and is therefore not a spectral set. It is shown that every nonnormal subnormal operator is the limit of a sequence of hyponormal and nonsubnormal operators.
\end{abstract}

1. Introduction. An operator $T$ on a Hilbert space $\mathfrak{H C}$ is said to be semi-normal in case its selfadjoint selfcommutator $T^{*} T-T T^{*}=D$ is positive semidefinite $(D \geqq 0)$ or negative semidefinite $(D \leqq 0)$. In the case $D \geqq 0$ the operator $T$ is called hyponormal. An interesting subclass of the hyponormal operators is the class of subnormals: an operator $T$ on $\mathcal{H C}$ is said to be subnormal in case $T$ is the restriction of a normal operator $A$ acting on a superspace $\Re \supset \mathcal{H}$.

It is known that the spectrum of a subnormal operator is a spectral set (see, e.g., Lebow [5]). Moreover, Bishop [2] has characterized the subnormal operators as precisely the closure, in the strong operator topology, of the normal operators (see also Stampfli [10]).

In this note an example is given of a seminormal operator whose spectrum is not a spectral set ( $\$ 3)$. This example motivates a construction which shows that every nonnormal subnormal operator is a strong limit of a sequence of hyponormal and nonsubnormal operators $(\$ 4)$.

2. Preliminaries. If $X$ is a compact set in the plane, then $C(X)$ will denote the algebra of all complex continuous functions on $X$ with norm defined by $\|f\|_{X}=\sup \{|f(z)|: z \in X\}$ for $f$ in $C(X)$. The symbols $R(X)$ and $P(X)$ will be used to denote, respectively, the set of restrictions to $X$ of the rational functions without poles in $X$ and the polynomial functions. The closures of $R(X)$ and $P(X)$ in $C(X)$ will be denoted by $\mathrm{Cl}(R(X))$ and $\mathrm{Cl}(P(X))$.

The spectrum of an operator $T$ is denoted by $\sigma(T)$. If $X$ is a set in the plane, $\operatorname{pr}_{x}(X)$ and $\operatorname{pr}_{y}(X)$ will be used to denote the projections of $X$ on the $x$ and $y$-axes. The notations meas md $_{1}$ aneas ${ }_{2}$ will be employed for linear and planar Lebesgue measure, respectively.

Received by the editors April 22, 1969.

AMS Subject Classifications. Primary 4615, 4710.

Key Words and Phrases. Seminormal operator, hyponormal operator, subnormal operator, spectral set. 
A compact set $X$ in the plane is called a spectral set for an operator $T$ in case $\|f(T)\| \leqq\|f\|_{X}$ for all $f \in R(X)$.

The following theorem of von Neumann $[7$, p. 279, Satz 6.5] is also proved in Lebow [5]:

TheOREM vN. If $\mathrm{Cl}(R(X))=C(X)$ and $X$ is a spectral set for the operator $T$, then $T$ is normal.

The following approximation theorem is due to Lavrentieff [4] (cf. Wermer [11, p. 74, Theorem 7.3] and Rudin [9, p. 386]):

Theorem L. If $X$ is a compact set in the plane having no interior and such that the complement of $X$ is connected, then $\mathrm{Cl}(P(X))=C(X)$.

The next two theorems appear in Putnam [8, p. 46 and p. 54]:

Theorem P1. If $T=H+i J$ is the Cartesian decomposition of the seminormal operator $T$, then $\operatorname{pr}_{x}(\sigma(T))=\sigma(H)$ and $\operatorname{pr}_{y}(\sigma(T))=\sigma(J)$.

THEOREM P2. If $T=H+i J$ is seminormal and $\sigma(H)$ contains no interval, then $\left\|T^{*} T-T T^{*}\right\| \leqq(1 / \pi)$ meas $_{2} \sigma(T)$.

An operator $T$ is called normaloid if $\sup \{|\lambda|: \lambda \in \sigma(T)\}=\|T\|$.

Berberian [1] has given the following characterization of operators whose spectrum is a spectral set:

Theorem B. The spectrum $\sigma(T)$ is a spectral set for the operator $T$ if and only if $f(T)$ is normaloid for every $f \in R(\sigma(T))$.

Finally, if $H$ is selfadjoint, with spectral resolution $H=\int \lambda d E_{\lambda}$, then $H$ is said to be absolutely continuous in case $\left\|E_{\lambda} x\right\|^{2}$ is an absolutely continuous function of $\lambda$, for each $x \in H$.

3. The example. Kato [3] has given examples of seminormal operators whose real parts have spectra that are Cantor sets of positive measure. The following example is of the type studied by Kato.

Let $K$ be a bounded real Cantor set of positive measure; such a set $K$ is perfect in measure, that is, every neighborhood of a point in $K$ intersects $K$ in a set of positive measure. For $f \in L^{2}(K)$ (with respect to Lebesgue measure), define the operator

$$
T f(s)=s f(s)+(1 / \pi) \int_{K} f(t)(s-t)^{-1} d t \quad(s \in K) .
$$

If the singular integral is interpreted as a Cauchy principal value, then the operator $T$ is bounded (see, e.g., Mushelišvili [6]). Moreover, $\left(T^{*} T-T T^{*}\right) f=(2 / \pi)\left(f, 1_{K}\right) 1_{K}$, where $1_{K}$ denotes the characteristic 
function of $K$, so that $T$ is hyponormal and nonnormal. Clearly $\left\|T^{*} T-T T^{*}\right\|=(2 / \pi)$ meas $_{1} K$.

The selfadjoint operators $H$ and $J$, defined by $H f(s)=s f(s)$ and $J f(s)=(1 / \pi i) \int_{K} f(t)(s-t)^{-1} d t$ for $s \in K$ and $f \in L^{2}(K)$, are the real and imaginary parts of $T$, respectively. It is known that $\sigma(H)=K$, and that $\sigma(J)=[-1,1]$ (see, e.g., Putnam [8, p. 140]); it follows from Theorem P1 that $\sigma(T) \subset K \times[-1,1]$, therefore meas $2 \sigma(T)$ $\leqq 2$ meas $_{1} K$. On the other hand, since $\left\|T^{*} T-T T^{*}\right\|=(2 / \pi)$ meas $_{1} K$, Theorem P2 yields 2 meas $_{1} K \leqq$ meas $_{2} \sigma(T)$. Thus meas $2 \sigma(T)$ $=2$ meas $_{1} K=\operatorname{meas}_{2}(K \times[-1,1])$. Since $K$ is perfect in measure one easily concludes that $\sigma(T)=K \times[-1,1]$.

Then $\sigma(T)$ is a compact set in the plane without interior and with connected complement, hence by Theorem $\mathrm{L} \mathrm{Cl}(P(\sigma(T)))$ $=\mathrm{Cl}(R(\sigma(T)))=C(\sigma(T))$. It follows that $\sigma(T)$ is not a spectral set for $T$ (if it were, Theorem vN would imply $T$ normal). The operator $T$ cannot be subnormal since the spectrum of a subnormal operator is a spectral set [5, p. 82].

From Theorem B and a lemma of Lebow $[5$, p. 66] an additional interesting property of our example $T$ can be concluded: for some polynomial $p$ the operator $p(T)$ is nonnormaloid.

4. The construction. Let $T=H+i J$ be hyponormal and nonnormal, so that $\left(D x_{0}, x_{0}\right)>0$ for some $x_{0}$ in $\Re$. Assume $H$ is absolutely continuous with spectral measure $E(\beta)$ for $\beta$ a Borel set.

Choose an increasing sequence $\left\{\beta_{n}\right\}$ of perfect nowhere dense sets in $\sigma(H)$ such that meas $_{1}\left(\beta_{n}\right) \rightarrow$ meas $_{1}(\sigma(H))$. Then since $H$ is absolutely continuous, $E\left(\beta_{n}\right) x \rightarrow x$ for all $x$ in $\mathcal{H}$. From the identity $D=2 i[H J-J H]$ it easily follows that the operators $T_{\beta}=E(\beta) T E(\beta)$ are hyponormal on $\mathcal{H C}$. Moreover, $\lim T_{\beta_{n}} x=T x$ for all $x$. Further for $n$ sufficiently large $\left(E\left(\beta_{n}\right) D E\left(\beta_{n}\right) x_{0}, x_{0}\right)$ must be positive. Since $T_{\beta_{n}}^{*} T_{\beta_{n}}$ $-T_{\beta_{n}} T_{\beta_{n}}^{*}=E\left(\beta_{n}\right) D E\left(\beta_{n}\right)$, then for large $n, T_{\beta_{n}}$ will be hyponormal and nonnormal. It follows from Theorem P1 that $\sigma\left(T_{\beta_{n}}\right) \subseteq$ $\beta_{n} \times[-1,1] \cup\{0\}$.

As in $\S 3$ it is possible to conclude, when $n$ is large, that $\sigma\left(T_{\beta_{n}}\right)$ is not a spectral set for $T_{\beta_{n}}$ and hence $T_{\beta_{n}}$ is hyponormal and nonsubnormal.

The requirement that $H$ be absolutely continuous can be removed in virtue of the following result of Putnam [8, p. 42]:

Theorem P3. Let $T=H+i J$ be hyponormal. Let $\mathfrak{T}=\mathfrak{N}_{T}$ denote the smallest subspace of $H$ which is invariant under $T$ and $T^{*}$ and contains the range of $D=T^{*} T-T T^{*}$. Then $\mathfrak{M}$ reduces $T$ and if $T^{\prime}=H^{\prime}+i J^{\prime}$ is 
the restriction of $T$ to $\mathrm{TT}$ then $H^{\prime}$ is absolutely continuous. Moreover, $T$ on the orthogonal complement of $\mathfrak{T C}$ is a normal operator.

From the above construction and Theorem P3 there follows:

Theorem C. Let $T$ be subnormal and nonnormal. Then $T$ is the strong limit of a sequence of hyponormal and nonsubnormal operators.

Acknowledgement. The results in this paper form a portion of the author's doctoral dissertation prepared under the direction of Professor C. R. Putnam at Purdue University.

\section{REFERENCES}

1. S. K. Berberian, A note on operators whose spectrum is a spectral set, Acta. Sci. Math. (Szeged) 27 (1966), 201-203. MR 34 \#3309.

2. E. Bishop, Spectral theory for operators on a Banach space, Trans. Amer. Math. Soc. 86 (1957), 414-445. MR $20 \# 7217$.

3. T. Kato, Smooth operators and commutators, Studia Math. 31 (1968), 535-546. MR $38 \# 2631$.

4. M. Lavrentieff, Sur les fonctions d'une variable complexe représentables par des séries de polynomes, Actualités Sci. Indust., no. 441, Hermann, Paris, 1936.

5. A. Lebow, On von Neumann's theory of spectral sets, J. Math. Anal. Appl. 7 (1963), 64-90. MR 27 \#6149.

6. N. I. Mushelišvili, Singular integral equations. Boundary problems of function theory and their application to mathematical physics, OGIZ, Moscow, 1946; English transl., Noordhoff, Groningen, 1953. MR 8, 586; MR 15, 434.

7. J. von Neumann, Eine Spektraltheorie für allgemeine Operatoren eines unitären Raumes, Math. Nachr. 4 (1951), 258-281. MR 13, 254.

8. C. R. Putnam, Commutation properties of Hilbert space operators and related topics, Ergebnisse der Math. und ihrer Grenzgebiete, Band 36, Springer-Verlag, New York, 1967. MR $36 \# 707$.

9. W. Rudin, Real and complex analysis, McGraw-Hill, New York, 1966. MR 35 $\# 1420$.

10. J. G. Stampfli, On operators related to normal operators, Ph.D. Thesis, University of Michigan, Ann Arbor, Mich., 1959.

11. J. Wermer, Banach algebras and analytic functions, Advances in Math. 1 (1961), no. 1, 51-102. MR 26 \#629.

Carleton University 